\title{
COMPORTAMIENTOS SEXUALES DE RIESGO EN ADOLESCENTES DEL SISTEMA DE PROTECCIÓN: UNA REVISIÓN SISTEMÁTICA
}

\author{
Olga Fernández-García \\ Dpto. de Psicología Evolutiva y de la Educación, Universitat de València, Valencia, España \\ olga.fernandez-garcia@uv.es \\ Verónica Estruch-García \\ Dpto. de Psicología Evolutiva y de la Educación, Universitat de València, Valencia, España
}

vesgar2@alumni.uv.es

Cristina Giménez-García

Dpto. de Psicología Básica, Clínica y Psicobiología, Universitat Jaume I, Castellón, España gimenezc@uji.es Jesús Castro-Calvo

Dpto. de Personalidad, Evaluación y Tratamientos Psicológicos, Universitat de València, Valencia, España jesus.castro@uv.es

\author{
Rafael Ballester-Arnal \\ Dpto. de Psicología Básica, Clínica y Psicobiología, Universitat Jaume I, Castellón, España \\ rballest@psb.uji.es \\ María Dolores Gil-Llario
}

1Dpto. de Psicología Evolutiva y de la Educación, Universitat de València, Valencia, España dolores.gil@uv.es

Recepción Artículo: 27 octubre 2021 Admisión Evaluación: 27 octubre 2021 Informe Evaluador 1: 28 octubre 2021 Informe Evaluador 2: 29 octubre 2021 Aprobación Publicación: 30 octubre 2021

\section{RESUMEN}

La salud sexual de los/as adolescentes y jóvenes suele mirarse desde una lente que se enfoca en los problemas. En el caso de los adolescentes que están en el sistema de protección infantil este análisis centrado en los riesgos es mayor si cabe, dado que experimentan tasas más altas de resultados negativos de salud sexual. La presente revisión tiene el objetivo de examinar la literatura relacionada con los comportamientos sexuales de riesgo de los adolescentes y jóvenes que se encuentran en el sistema de protección. Para ello, se han consultado varias bases de datos (Web of Science, Scopus y PUBMED), restringiendo la búsqueda a las palabras clave "sexual behavior" o "sexual risk" y "child welfare system" o "foster youth", y el rango de publicación entre 2011 y 2021. De las 1496 publicaciones identificadas inicialmente, se eliminaron aquellas duplicadas y/o que no cumplían los criterios de inclusión, quedándonos con un total de 22 artículos. La mitad de las publicaciones que analizaban los comportamientos predictivos de conductas sexuales de riesgo se centraban en las experiencias de abuso sexual en la infancia. Por otro lado, el 22,7\% de los artículos analizados informaban de un uso deficitario 


\title{
COMPORTAMIENTOS SEXUALES DE RIESGO EN ADOLESCENTES DEL SISTEMA DE PROTECCIÓN: UNA REVISIÓN SISTEMÁTICA
}

del preservativo entre los jóvenes en hogares de acogida, mientras que el 18,2\% afirmaban que este colectivo presenta una mayor prevalencia de ITS y el 31,8\% reportan que las tasas de embarazo son más altas entre las chicas de esta población. Asimismo, el 18,2\% de los estudios analizados encontraron indicios de una posible relación entre la participación en el sistema de bienestar infantil y en actividades de sexo transaccional. Así, aunque la investigación sobre este tema sigue siendo escasa, esta revisión pone de manifiesto el riesgo de desarrollar patrones poco saludables de sexualidad entre los jóvenes del sistema de protección.

Palabras clave: revisión bibliográfica; comportamientos sexuales de riesgo; adolescentes; jóvenes; sistema de protección

\begin{abstract}
Sexual risk behaviors in adolescents in the child welfare system: A systematic review. The sexual health of adolescents and youth is often viewed through a lens that focuses on the problems. For adolescents in the child welfare system this risk-focused analysis is even greater, as they experience higher rates of negative sexual health outcomes. The present review aims to examine the literature related to sexual risk behaviors of adolescents and young people in the child welfare system. For this purpose, several databases (Web of Science, Scopus and PUBMED) were consulted, restricting the search to the keywords "sexual behavior" or "sexual risk" and "child welfare system" or "foster youth", and the publication range between 2011 and 2021. Of the 1496 publications initially identified, those that were duplicated and/or did not meet the inclusion criteria were eliminated, leaving us with a total of 22 articles. Half of the publications analyzing behaviors predictive of sexual risk behaviors focused on childhood sexual abuse experiences. On the other hand, $22.73 \%$ of the articles analyzed reported poor condom use among youth in foster care, while $18.18 \%$ stated that this group has a higher prevalence of STIs and $31.82 \%$ reported that pregnancy rates are higher among girls in this population. Likewise, $18.18 \%$ of the studies analyzed found evidence of a possible relationship between participation in the child welfare system and transactional sex activities. Thus, although research on this topic remains scarce, this review highlights the risk of developing unhealthy patterns of sexuality among youth in the child welfare system.
\end{abstract}

Keywords: literature review; sexual risk behaviors; adolescents; youth; child welfare system

\section{INTRODUCCIÓN}

Los conocimientos, habilidades y actitudes que el/la adolescente posee sobre sexualidad van a determinar que realice una correcta toma de decisiones a la hora de involucrarse en prácticas sexuales seguras y saludables (Diamant-Wilson y Blakey, 2019). De aquí el interés de los/as profesionales de esta área en que dicho grupo poblacional reciba la información necesaria para experimentar un desarrollo sexual adecuado (Voisin y Bird, 2009). Sin embargo, comúnmente la sexualidad de los/as adolescentes y jóvenes ha sido abordada desde un enfoque basado en los riesgos y en las conductas sexuales problemáticas. En el caso de los/as adolescentes y jóvenes inmersos en el sistema de protección esta consideración es aún mayor (Child Welfare Information Gateway, 2016; Diamant-Wilson y Blakey, 2019; Simkiss et al., 2012; Ramseyer et al., 2016).

Los/Las jóvenes que han pasado su minoría de edad en hogares de acogimiento residencial tuvieron que separarse de su familia al ver vulnerados sus derechos y quedar desprotegidos (Fernández-García et al., 2021). Es más, en la mayoría de las ocasiones, han sido víctimas de maltrato físico, emocional y/o sexual, y han crecido en un ambiente de inestabilidad familiar sin una figura de referencia estable y accesible (Tarren-Sweeney, 2008), factores de riesgo que aumentan la probabilidad de presentar resultados adversos en múltiples dominios de su vida y de participar en conductas de riesgo para la salud (Harden, 2004). Concretamente, respecto a su desarrollo sexual, Stott (2012), tras evaluar las conductas sexuales de riesgo en un grupo de jóvenes con una medida de acogimiento residencial, informó que el $47.2 \%$ se involucraba en bastantes comportamientos sexuales problemáticos y el $31.1 \%$ había realizado todos los comportamientos sexuales por los que se preguntaba. Un porcentaje realmente preocupante que algunos autores asocian con las dificultades que estos chicos/as pueden tener a la hora de acceder a servicios de planificación familiar al no recibir la información adecuada por parte de un refe- 
rente familiar. Pergamit y Johnson (2009) reportaron que sólo el 18\% de los jóvenes de acogida de su encuesta que habían sido sexualmente activos en el último año informaron que habían utilizado servicios de salud reproductiva durante el mismo período de tiempo.

En este afán por explicar la elevada toma de riesgos, algunas investigaciones vinculan la experiencia de abuso sexual con la participación en conductas sexuales de riesgo (Lalor y McElvaney, 2010), por lo que dada la alta prevalencia de este tipo de experiencias entre los niños/as y adolescentes involucrados en este sistema (Dorsey et al., 2012), no es de extrañar que este colectivo participe más frecuentemente en conductas sexuales de riesgo. En esta línea, el apoyo que la persona menor de edad ha recibido por parte de sus padres (Taussig, 2002), la implicación de sus iguales en conductas sexuales de riesgo también (Polgar y Auslander, 2009) y la experimentación de sintomatología traumática (Taussig y Talmi, 2001), también parecen ser factores facilitadores de conductas sexuales de riesgo.

Si realizamos un análisis más minucioso teniendo en cuenta los diferentes comportamientos sexuales que pueden ser problemáticos, encontramos estudios que se hacen eco de un mayor riesgo de embarazo precoz y una mayor incidencia de infecciones de transmisión sexual (ITS), incluyendo tasas más altas de VIH (Ahrens et al., 2013), entre los/as adolescentes y jóvenes del sistema de protección frente a sus iguales que no fueron separados de su familia biológica. Estos resultados se asocian con un menor uso de anticonceptivos en este grupo muestral, aunque los resultados no parecen ser concluyentes, ya que hay investigaciones que reportan porcentajes similares en todos/as los/as jóvenes independientemente de si han pasado su infancia en un hogar de acogimiento residencial o no (Courtney et al., 2010). Asimismo, aunque no hay demasiados estudios que exploren esta problemática, también se ha encontrado una participación más alta en actividades de sexo transaccional (intercambio de actividades sexuales por recursos) entre quienes crecen fuera del hogar familiar y, en mayor medida, si fueron víctimas de abuso sexual en la infancia (Ahrens et al., 2012, Surratt et al., 2004).

\section{OBJETIVOS DE LA INVESTIGACIÓN}

El conocimiento de los riesgos asociados a la salud sexual de los/as adolescentes y jóvenes que están o han estado inmersos en el sistema de protección infantil es realmente importante para los profesionales que trabajan diariamente con ellos/as, ya que, en parte, esto va a determinar que realicen una intervención consciente y adaptada a sus necesidades, ayudándoles a tener un desarrollo saludable.

Vinculado a esto, el presente trabajo tiene el objetivo de realizar una revisión sistemática en la que se examine la literatura más reciente relacionada con los comportamientos sexuales de riesgo de los/as adolescentes y jóvenes que se encuentran inmersos en el sistema de protección.

\section{METODOLOGíA}

Con el objetivo de garantizar la calidad de esta revisión sistemática se siguieron las directrices de la declaración PRISMA (Liberati et al., 2009) realizándose búsquedas en las bases de datos Web of Science, Scopus y PUBMED de los trabajos publicados en los últimos 10 años (2011-2021) con los siguientes descriptores: "sexual behavior", "sexual risk", "child welfare system" y "foster youth", combinados para dar lugar a las siguientes ecuaciones de búsqueda: ("sexual behavior" OR "sexual risk") AND ("child welfare system" OR "foster youth").

Para ser incluidos en esta revisión, los trabajos debían abordar los comportamientos sexuales de riesgo de los/as adolescentes o jóvenes inmersos/as en el sistema de protección. La búsqueda inicial identificó 1496 posibles publicaciones, de las cuales 979 se eliminaron al estar duplicados. Tras leer los títulos y resúmenes del resto de trabajos, 495 fueron eliminados por no cumplir con los criterios de inclusión: 241 por no centrarse en la población objeto de estudio (adolescentes y jóvenes del sistema de protección), 102 por no tratar sobre el desarrollo sexual y 152 porque, aunque abordaban este tema, no se centraban específicamente en el análisis de los comportamientos sexuales de riesgo. Por lo tanto, un total de 22 artículos pasaron a conformar la muestra definitiva de este estudio. En la Fig. 1 se incluye el diagrama de flujo de la estrategia metodológica seguida en la selección de los artículos. 
Fig. 1. Diagrama de flujo de la estrategia metodológica seguida en la revisión sistemática

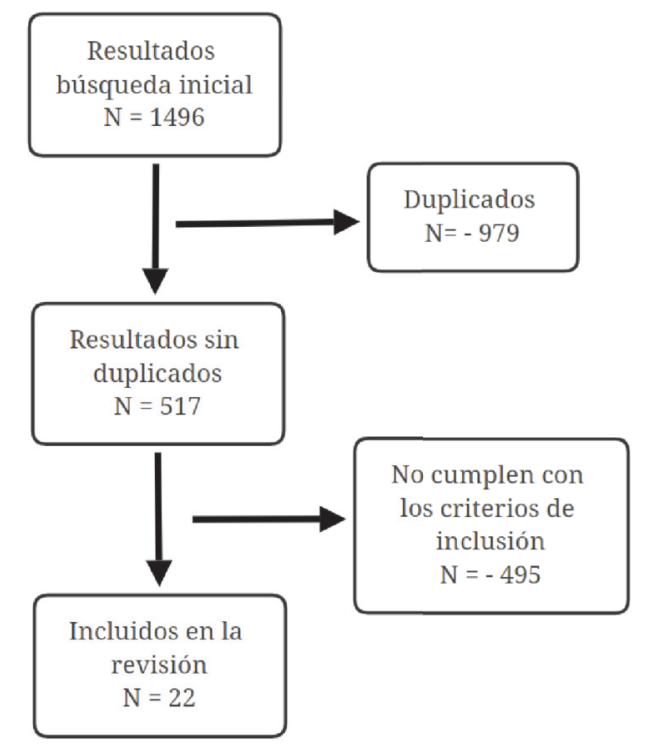

\section{RESULTADOS ALCANZADOS}

La literatura encontrada, además de informar acerca de los factores asociados a la toma de riesgos sexuales, diferencia entre distintos comportamientos sexuales problemáticos. Así, para la exposición de los resultados se han establecido categorías en función de los diferentes comportamientos sexuales de riesgo de los que hablan los artículos encontrados: factores predictivos, uso de anticonceptivos, prevalencia e incidencia de embarazo y de ITS, y sexo transaccional. En la tabla 2 se muestran las características principales de las publicaciones examinadas.

\section{Factores predictivos}

El 40,9\% de las publicaciones encontradas analizaban la influencia que ciertos factores (experiencias, vivencias, etc.) tienen en la participación en comportamientos sexuales de riesgo.

Mientras el 55,5\% de los artículos que hablan sobre los factores predictivos muestran una relación positiva entre el abuso sexual en la infancia y las conductas sexuales de riesgo [1,2, 10, 12, 21], el 22,22\% exploró cómo la relación que los adolescentes y jóvenes con una medida de acogimiento residencial tienen con sus familias biológicas influye en la toma de riesgos sexuales [10, 14]. En este sentido, Maliszewski y Brown (2014) encontraron que la relación positiva entre los jóvenes en acogida y sus hermanos biológicos se correlaciona con la predisposición a practicar sexo seguro. Asimismo, González-Blanks y Yates (2015) concluyen que los intentos fallidos de reunificación familiar se asocian con mayor incurrencia de conductas sexuales de riesgo.

Otro 22,2\% de los artículos encontraron una relación positiva entre el desarrollo de síntomas de estrés postraumático o disociativos y la probabilidad de implicarse en relaciones sexuales sin protección $[4,10]$. Sin embargo, únicamente se ha encontrado un artículo que confirme que los comportamientos delictivos y el consumo de alcohol y marihuana son variables predictivas de la participación en conductas de riesgo de VIH [20].

Por último, Fowler et al. (2014) tras examinar los cambios en las conductas sexuales de riesgo a largo plazo en los/as jóvenes y adolescentes del sistema de protección, determinó que ninguno de los factores del entorno 
que otros estudios habían analizado (abuso sexual, delincuencia, abuso de sustancias psicoactivas) podían ser considerados predictores de las conductas sexuales de riesgo.

TABLA 1. Distribución de las publicaciones en función de los factores predictivos que analizan

\begin{tabular}{lcllll}
\hline & $\begin{array}{l}\text { Vivencia } \\
\text { abuso } \\
\text { sexual }\end{array}$ & $\begin{array}{l}\text { Proble } \\
\text { mas } \\
\text { salud } \\
\text { mental } \\
\text { delictivos y } \\
\text { consumo } \\
\text { sust. } \\
\text { psicoactivas }\end{array}$ & $\begin{array}{l}\text { Relación } \\
\text { familia } \\
\text { biológica }\end{array}$ & $\begin{array}{l}\text { No } \\
\text { influencia } \\
\text { factores } \\
\text { del } \\
\text { entorno }\end{array}$ \\
\hline Ahrens et al. (2012) & $\mathrm{X}$ & & & & \\
\hline $\begin{array}{l}\text { Brandon-Friedman } \\
\text { et al. (2020) }\end{array}$ & $\mathrm{X}$ & & & $\mathrm{X}$ \\
\hline Cavanaugh (2013) & & $\mathrm{X}$ & & $\mathrm{X}$ \\
\hline Fowler et al. (2014) & & & & $\mathrm{X}$ \\
\hline $\begin{array}{l}\text { González-Blanks y } \\
\text { Yates (2015) }\end{array}$ & $\mathrm{X}$ & $\mathrm{X}$ & & \\
\hline Grossi et al. (2016) & $\mathrm{X}$ & & & & \\
\hline $\begin{array}{l}\text { Maliszewski y } \\
\text { Brown (2014) }\end{array}$ & & & & & \\
\hline $\begin{array}{l}\text { Thompson y } \\
\text { Auslander (2011) }\end{array}$ & & & & $\mathrm{X}$ & \\
\hline Wekerle et al. (2017) & $\mathrm{X}$ & & & & \\
\hline
\end{tabular}

\section{Uso del preservativo}

Respecto al uso de métodos anticonceptivos, el $18,2 \%$ de las publicaciones exploran la frecuencia de uso del preservativo entre los/as adolescentes y jóvenes del sistema de protección $[5,8,9,18]$, y concluyen que más del $45 \%$ de su muestra no usa el preservativo cada vez que mantienen relaciones sexuales, Ilegando hasta casi el $60 \%$ de prevalencia cuando se pregunta si han mantenido relaciones sin preservativo alguna vez en los últimos 3 meses. En esta línea, Fowler, et al. (2014) encontraron que sólo el 10\% de los jóvenes que encuestaron habían usado el preservativo durante su relación sexual más reciente.

\section{Prevalencia e incidencia de ITS}

Cinco de las publicaciones encontradas informan sobre las tasas de ITS de los/as adolescentes y jóvenes del sistema de protección $[1,6,17,18,19]$. En este sentido, aunque la mayoría (80\%) de estos artículos reportan una incidencia mayor de ITS en los/as adolescentes y jóvenes que están en hogares de acogimiento residencial frente a los que no lo están $[1,6,17,19]$, el 20\% no encontró una relación significativa entre la inestabilidad de colocación que caracteriza a este colectivo y esta mayor incidencia [18]. Asimismo, las tasas de incidencia son algo dispares ya que, mientras Stott (2012) concluyó que el 24\% de los encuestados aseguraban haber tenido alguna vez una ITS, Courtney et al. (2011) informaban de una incidencia del 3,8\% en mujeres y del 19,1\% en hombres.

\section{Prevalencia e incidencia de embarazos}

De los siete artículos que exploran la prevalencia de embarazos entre las jóvenes con una medida de acogimiento residencial $[3,7,13,15,16,18,22]$, el $100 \%$ reportó que estas tenían mayor probabilidad de quedarse embarazadas que sus homólogas que no estaban ni habían estado en hogares de acogida. En esta línea, el 71,4\% de estos artículos informaron de tasas de embarazo que estaban por encima del $40 \%$ en grupos de chicas de entre 18 y 21 años que vivían en hogares de acogimiento residencial [3, 15, 16, 18, 22]. Sin embargo, solo el 42,9\% 
de los artículos compararon estas tasas con las de sus iguales que no estaban en el sistema de protección, informando que, aunque la prevalencia era mayor en este colectivo, la diferencia es modesta $[7,13,18]$.

\section{Sexo transaccional}

De las 22 publicaciones que hemos analizado en este estudio, el 18,2\% exploraban la participación de este colectivo en experiencias de sexo transaccional, y todas ellas concluyen que hay indicios de una posible relación entre la pertenencia al sistema de protección y la realización de actividades sexuales a cambio de una compensación económica [1, 6, 11, 19]. Ahrens et al. (2012) se aventuran a demostrar el papel del acoso sexual y/o violación como variables mediadoras de esta relación.

TABLA 2. Características principales de los estudios incluidos

\begin{tabular}{|c|c|c|c|}
\hline $\mathbf{N}^{\mathbf{0}}$ & Autor(es) (año) & $\begin{array}{l}\text { Características de la muestra } \\
\text { (N/Edad) }\end{array}$ & $\begin{array}{l}\text { Comportamientos sexuales de } \\
\text { riesgo considerados }\end{array}$ \\
\hline 1 & Ahrens et al. (2012) & $\mathrm{N}=574 ; \mathrm{M}$ edad $=17$ & $\begin{array}{l}\text { Factores predictivos de comp. } \\
\text { sexuales de riesgo } \\
\text { ITS } \\
\text { Sexo transaccional }\end{array}$ \\
\hline 2 & $\begin{array}{l}\text { Brandon-Friedman et } \\
\text { al. (2020) }\end{array}$ & $\mathrm{N}=219 ; \mathrm{Edad}=18-24$ años & $\begin{array}{l}\text { Factores predictivos de comp. } \\
\text { sexuales de riesgo }\end{array}$ \\
\hline 3 & $\begin{array}{l}\text { Casanueva et al. } \\
(2014)\end{array}$ & $\mathrm{N}=615 ; \mathrm{Edad}=11-20$ años & Embarazos \\
\hline 4 & Cavanaugh (2013) & $\mathrm{N}=285 ; \mathrm{M}$ edad $=13.7$ & $\begin{array}{l}\text { Factores predictivos de comp. } \\
\text { sexuales de riesgo }\end{array}$ \\
\hline 5 & Cheung et al. (2014) & $\mathrm{N}=437 ; \mathrm{M}$ edad $=15$ & Uso del preservativo \\
\hline 6 & Courtney et al. (2011) & $\mathrm{N}=596 ; \mathrm{M}$ edad $=26$ & $\begin{array}{l}\text { ITS } \\
\text { Sexo transaccional }\end{array}$ \\
\hline 7 & Courtney et al. (2014) & $\mathrm{N}=727 ; \mathrm{M}$ edad $=16.96$ & Embarazos \\
\hline 8 & $\begin{array}{l}\text { Finigan-Carr et al. } \\
(2021)\end{array}$ & $\begin{array}{l}\mathrm{N}=318 \text { hombres; } \mathrm{Edad}=13-21 \\
\text { años }(\mathrm{M}=17.9)\end{array}$ & Uso del preservativo \\
\hline 9 & Fowler et al. (2014) & $\mathrm{N}=714 ; \mathrm{M}$ edad $=15.5$ & $\begin{array}{l}\text { Factores predictivos de comp. } \\
\text { sexuales de riesgo } \\
\text { Uso del preservativo }\end{array}$ \\
\hline 10 & $\begin{array}{l}\text { González-Blanks y } \\
\text { Yates (2015) }\end{array}$ & $\mathrm{N}=118 ; \mathrm{M}$ edad $=19.61$ & $\begin{array}{l}\text { Factores predictivos de comp. } \\
\text { sexuales de riesgo }\end{array}$ \\
\hline 11 & Greeson et al. (2019) & $\mathrm{N}=270 ; \mathrm{M}$ edad $=20.7$ & Sexo transaccional \\
\hline 12 & Grossi et al. (2016) & $\begin{array}{l}\mathrm{N}=250 ; \\
\mathrm{M} \text { edad mujeres }=9.52 \\
\mathrm{M} \text { edad mujeres }=9.76\end{array}$ & $\begin{array}{l}\text { Factores predictivos de comp. } \\
\text { sexuales de riesgo }\end{array}$ \\
\hline 13 & King et al. (2014) & $\begin{array}{l}\mathrm{N}=\text { varía por año; Edad }=15-17 \\
\text { años }\end{array}$ & Embarazos \\
\hline 14 & $\begin{array}{l}\text { Maliszewski y Brown } \\
(2014)\end{array}$ & $\mathrm{N}=100 ; \mathrm{M}$ edad $=21.94$ & $\begin{array}{l}\text { Factores predictivos de comp. } \\
\text { sexuales de riesgo }\end{array}$ \\
\hline 15 & Oshima et al. (2013) & $\mathrm{N}=325 ; \mathrm{Edad}=19$ años & Embarazos \\
\hline 16 & PettyJohn et al. (2021) & $\begin{array}{l}\mathrm{N}=136 \text { mujeres; Edad =6-24 } \\
\text { años }\end{array}$ & Embarazos \\
\hline 17 & Robertson (2013) & - & ITS \\
\hline 18 & Stott (2012) & $\mathrm{N}=114 ; \mathrm{Edad}=18-21$ años & $\begin{array}{l}\text { Uso del preservativo } \\
\text { ITS } \\
\text { Embarazos }\end{array}$ \\
\hline 19 & Surratt y Kurtz (2012) & $\mathrm{N}=562$ mujeres; $\mathrm{M}$ edad $=35.5$ & $\begin{array}{l}\text { ITS } \\
\text { Sexo transaccional }\end{array}$ \\
\hline 20 & $\begin{array}{l}\text { Thompson y } \\
\text { Auslander (2011) }\end{array}$ & $\mathrm{N}=320 ; \mathrm{M}$ edad $=16.3$ & $\begin{array}{l}\text { Factores predictivos de comp. } \\
\text { sexuales de riesgo }\end{array}$ \\
\hline 21 & Wekerle et al. (2017) & $\mathrm{N}=297 ; \mathrm{M}$ edad $=16.3$ & $\begin{array}{l}\text { Factores predictivos de comp. } \\
\text { sexuales de riesgo }\end{array}$ \\
\hline 22 & Wilson et al. (2014) & $\mathrm{N}=710 ;$ Edad $\geq 11$ años & Embarazos \\
\hline
\end{tabular}




\section{DISCUSIÓN}

En general, la literatura publicada en los últimos 10 años sugiere que los/las adolescentes y jóvenes inmersos en el sistema de protección infantil presentan una mayor propensión a participar en comportamientos sexuales de riesgo.

En este sentido, respecto a los factores que podrían estar explicando esta mayor prevalencia, las experiencias de abuso sexual parece estar a la cabeza. En nuestro estudio, más de la mitad de los artículos revisados concluían la existencia de una relación positiva entre las experiencias de abuso sexual infantil y la participación en conductas sexuales de riesgo, aunque esta asociación podría variar en función de la historia de abuso (características del abuso, de la víctima y del perpetrador) (Ramseyer et al., 2016). Estudios anteriores también apoyaron esta relación (Paolucci et al., 2001, Van Roode et al., 2009). Asimismo, se ha encontrado literatura que apoya la influencia que las relaciones familiares, la presencia de problemas de salud mental y la participación en actividades delictivas y abuso de sustancias psicoactivas ejercen sobre la toma de riesgos sexuales. Sin embargo, no todos los estudios extraen estas conclusiones, ni ofrecen comparaciones directas entre los comportamientos de los/as jóvenes que están en hogares de acogida y sus compañeros no implicados en el sistema de protección, lo que justifica una mayor investigación en esta área y la interpretación cuidadosa de los resultados reportados.

Por otro lado, el uso del preservativo entre los/as jóvenes en acogimiento residencial parece insuficiente si tenemos en cuenta la información extraída de las investigaciones exploradas que reportan tasas de uso inferiores al 50\% entre los/as adolescentes de este colectivo. Sin embargo, a la hora de generalizar estos resultados, hay que tener en cuenta el reducido conjunto de investigaciones analizadas y las limitaciones que presentan estas al no recoger las características contextuales del participante (tipo relación, duración de la misma, etc.). Asimismo, investigaciones más antiguas reportaron tasas mucho más altas del uso del preservativo entre jóvenes del sistema de protección (Pergamit y Johnson, 2009), lo que contrasta con nuestras conclusiones.

El uso correcto y consistente de los métodos anticonceptivos es un componente de la salud sexual que se relaciona de forma directa con la prevalencia de embarazos y de ITS. Al analizar las tasas de ITS, vemos que estas varían en función del estudio y que son elevadas para todos/as los/las adolescentes independientemente de su implicación o no en el sistema de protección. Sin embargo, sí que parece aumentar de forma significativa el contagio durante y después de su estancia en el sistema de protección, lo que dispara las alertas de los profesionales de los hogares de acogimiento residencial. En investigaciones más antiguas, Pergamit y Johnson (2009) Ilegaron a conclusiones semejantes y Auslander et al. (2002) descubrieron que la experiencia de abuso sexual previo correlacionaba con las conductas de riesgo de VIH en este colectivo. Por otro lado, la literatura analizada sugiere que los/las jóvenes que están o han estado bajo la tutela de la administración pública pueden tener un mayor riesgo de embarazo. Estos resultados ya fueron encontrados en estudios anteriores, que además aseguraban la existencia de variaciones en las tasas de embarazo en función de la raza de la joven (Dworsky y Courtney, 2010; Pergamit y Johnson, 2009). Sin embargo, Ios artículos publicados sobre este tema no permiten extraer conclusiones generalizables respecto a las razones de esta alta prevalencia. Aunque frecuentemente se piensa que los embarazos a una edad temprana no son planificados, no siempre es así. En ocasiones, las jóvenes en acogida ven la maternidad como una oportunidad para demostrar su independencia y para desarrollar una nueva identidad proyectando sus deseos en sus hijos/as (Aparicio et al., 2015; Boustani et al., 2015; Connolly et al., 2012).

Por último, en relación al sexo transaccional, la literatura analizada va en la línea del resto de aspectos explorados. Los estudios informan de la mayor participación de los/las adolescentes y jóvenes que están o han estado en el sistema de protección en este tipo de actividades, frente a los que viven en el hogar familiar. Unos resultados realmente preocupantes si tenemos en cuenta que involucrarse en estas actividades puede conllevar resultados negativos de salud sexual (tasas más altas de ITS y de embarazos no deseados) (Falasinnu et al., 2014). Así, resulta de especial interés seguir investigando sobre este tema con el objetivo de alcanzar una mayor y mejor comprensión de los factores de riesgo y protección que contribuyen a la implicación en el sexo transaccional, así como las razones que llevan a estos jóvenes realizar actividades de este tipo (Ramseyer et al., 2016). 


\section{COMPORTAMIENTOS SEXUALES DE RIESGO EN ADOLESCENTES DEL SISTEMA DE PROTECCIÓN: UNA REVISIÓN SISTEMÁTICA}

\section{CONCLUSIONES}

Esta revisión revela la existencia de una base bibliográfica emergente sobre la salud sexual de los/las adolescentes y jóvenes que están o han estado en el sistema de protección infantil, la cual destaca el riesgo que estos/as presentan de experimentar resultados negativos en su desarrollo sexual afectando a su transcurso a la edad adulta. Por ello, tal y como lo hacen Boonstra (2011) y Geiger y Schelbe (2014), nos gustaría incidir en la importancia de asegurar una educación sexual integral que se centre realmente en las necesidades específicas de los/las niños/as y adolescentes que se encuentran en hogares de acogimiento residencial y que les aporte los conocimientos, actitudes y habilidades necesarias para realizar una toma de decisiones consciente. Además, dicha formación debe proporcionarse a una edad adecuada, teniendo en cuenta el inicio más temprano de este colectivo en las actividades sexuales, y garantizar su acceso a una atención sanitaria reproductiva adecuada, lo que pasa por la formación y el cambio de actitud de los profesionales que trabajan diariamente con ellos y que son quienes deben asegurarles un desarrollo saludable.

\section{REFERENCIAS BIBLIOGRÁFICAS}

Ahrens, K. R., Katon, W., McCarty, C., Richardson, L. P., \& Courtney, M. E. (2012). Association between childhood sexual abuse and transactional sex in youth aging out of foster care. Child Abuse and Neglect, 36(1), 75-80. http://dx.doi.org/10.1016/j.chiabu.2011.07.009

Ahrens, K. R., McCarty, C., Simoni, J., Dworsky, A., \& Courtney, M. E. (2013). Psychosocial pathways to sexually transmitted infection risk among youth transitioning out of foster care: Evidence from a longitudinal cohort study. Journal of Adolescent Health, 53(4), 478-485. http://dx.doi.org/10.1016/j.jadohealth.2013.05.010

Aparicio, E., Pecukonis, E. V., \& O'Neale, S. (2015). "The love that I wasmissing": Exploring the lived experience of motherhood among teen mothers in foster care. Children and Youth Services Review, 51, 44-54. http://dx.doi.org/10.1016/j.childyouth.2015.02.002

Auslander, W. F., McMillen, J. C., Elze, D., Thompson, R., Jonson-Reid, M., \& Stiffman, A. (2002). Mental health problems and sexual abuse among adolescents in foster care: Relationship to HIV risk behaviors and intentions. AIDS and Behavior, 6(4), 351-359. http://dx.doi.org/10.1023/a:1021152813774.

Boonstra, H. D. (2011). Teen pregnancy among young women in foster care: A primer. Guttmacher Policy Review, 14(2), 8-19.

Boustani, M. M., Frazier, S. L., Hartley, C., Meinzer, M., \& Hedemann, E. (2015). Perceived benefits and proposed solutions for teen pregnancy: Qualitative interviews with youth care workers. American Journal of Orthopsychiatry, 85(1), 80-92. http://dx.doi.org/10.1037/ort0000040

Brandon-Friedman, R. A., Wahler, E. A., Pierce, B. J., Thigpen, J. W., \& Fortenberry, J. D. (2020). The impact of sociosexualization and sexual identity development on the sexual well-being of youth formerly in the foster care system. Journal of Adolescent Health, 66(4), 439-446.

http://dx.doi.org/10.1016/j.jadohealth.2019.10.025

Casanueva, C., Tueller, S. J., Smith, K., Dolan, M., \& Ringeisen, H. (2014). NSCAW II Wave 3 report: Wave 3 tables final report. Washington, DC: Office of Planning, Research and Evaluation, Administration for Children and Families, US Department of Health and Human Services.

Cavanaugh, C. E. (2013). Brief report: The influence of posttraumatic stress on unprotected sex among sexually active adolescent girls and boys involved in the child welfare system of the United States. Journal of Adolescence, 36(5), 835 -837. http://dx.doi.org/10.1016/j.adolescence.2013.07.004

Cheung, K. K., Montgomery, D., \& Benjamins, L. J. (2014). Prevalence of sexually transmitted infections among adolescents entering child protective services. Journal of Pediatric and Adolescent Gynecology, 28(5), 324326. http://dx.doi.org/10.1016/j.jpag.2014.09.011

Child Welfare Information Gateway (2016). Foster care statistics 2014. Washington, D. C. Retrieved from https://www.childwelfare.gov/pubs/factsheets/foster/ 
Connolly, J., Heifetz, M., \& Bohr, Y. (2012). Pregnancy and motherhood among adolescent girls in child protective services: A meta-synthesis of qualitative research. Journal of Public Child Welfare, 6(5), 614-635. http://dx.doi.org/10.1080/15548732.2012.723970

Courtney, M. E., Charles, P., Okpych, N. J., Napolitano, L., \& Halsted, K. (2014). Findings from the California Youth Transitions to Adulthood Study (CalYOUTH): Conditions of foster youth at age 17. Chicago: Chapin Hall at the University of Chicago.

Courtney, M. E., Dworsky, A., Brown, A., Cary, C., Love, K., \& Vorhies, V. (2011). Midwest evaluation of the adult functioning of former foster youth: Outcomes at age 26. Chicago: Chapin Hall at the University of Chicago.

Courtney, M. E., Dworsky, A., Lee, J. S., \& Raap, M. (2010). Midwest evaluation of the adult functioning of former foster youth: Outcomes at ages 23 and 24. Retrieved from http://www.chapinhall.org/sites/default/files/Midwest_Study_Age_23_24.pdf

Diamant-Wilson, R., \& Blakey, J. M. (2019). "Strap up:" Sexual socialization and safer sex practices among African American youth in foster care. Child Abuse \& Neglect, 88, 466 - 477. http://dx.doi.org/10.1016/j.chiabu.2018.08.007

Dorsey, S., Burns, B. J., Southerland, D. G., Cox, J. R.,Wagner, H. R., \& Farmer, E. M. (2012). Prior trauma exposure for youth in treatment foster care. Journal of Child and Family Studies, 21(5), 816-824. http://dx.doi.org/10.1007/s10826-011-9542-4

Dworsky, A., \& Courtney,M. E. (2010). The risk of teenage pregnancy among transitioning foster youth: Implications for extending state care beyond age 18. Children and Youth Services Review, 32(10), 13511356. http://dx.doi.org/10.1016/j.childyouth.2010.06.002

Falasinnu, T., Gilbert,M., Salway, T. H., Gustafson, P., Ogilvie, G., \& Shoveller, J. (2014). Predictors identifying those at increased risk for STDs: A theory-guided review of empirical literature and clinical guidelines. International Journal of STD \& AIDS. http://dx.doi.org/10.1177/0956462414555930

Fernández-García, 0., Gil-Juliá, B., \& Gil-Llario, M. D. (2021). Salud sexual de los menores y jóvenes del sistema de protección y en conflicto con la ley. Revista INFAD de Psicología, 3(1).

https://doi.org/10.17060/ijodaep.2021.n1.v3.2028

Finigan-Carr, N. M., Craddock, J. B., \& Johnson, T. (2021). Predictors of Condom Use among System-Involved Youth: The Importance of Sex Ed. Children and Youth Services Review. https://doi.org/10.1016/j.childyouth.2021.106130

Fowler, P. J., Motley, D., Zhang, J., Rolls-Reutz, J., \& Landsverk, J. (2014). Adolescent maltreatment in the child welfare system and developmental patterns of sexual risk behaviors. Child maltreatment, 20(1), 50-60. http://dx.doi.org/10.1177/1077559514548701

Geiger, J. M., \& Schelbe, L. A. (2014). Stopping the cycle of child abuse and neglect: A call to action to focus on pregnant and parenting youth in and aging out of the foster care system. Journal of Public Child Welfare, 8(1), 25-50. http://dx.doi.org/10.1080/15548732.2013.824398

Gonzalez-Blanks, A., \& Yates, T. M. (2015). Sexual risk-taking among recently emancipated female foster youth: Sexual trauma and failed family reunification experiences. Journal of Research on Adolescence. 26(4), 819 - 829. http://dx.doi.org/10.1111/jora.12232

Greeson, J. K., Treglia, D., Wolfe, D. S., Wasch, S., \& Gelles, R. J. (2019). Child welfare characteristics in a sample of youth involved in commercial sex: An exploratory study. Child abuse \& neglect, 94. https://doi.org/10.1016/j.chiabu.2019.104038

Grossi, L. M., Lee, A. F., Schuler, A., Ryan, J. L., \& Prentky, R. A. (2016). Sexualized behaviors in cohorts of children in the child welfare system. Child abuse \& neglect, 52, $49-61$. https://doi.org/10.1016/j.chiabu.2015.12.014

Harden B. (2004). Safety and stability for foster children. The Future of Children, 14(1), 30 - 47. https://doi.org/10.2307/1602753 
King, B., Putnam-Hornstein, E., Cederbaum, J. A., \& Needell, B. (2014). A cross-sectional examination of birth rates among adolescent girls in foster care. Children and Youth Services Review, 36, 179-186. http://dx.doi.org/10.1016/j.childyouth.2013.11.007

Lalor, K., \& McElvaney, R. (2010). Child sexual abuse, links to later sexual exploitation/high-risk sexual behavior, and prevention/treatment programs. Trauma, Violence \& Abuse, 11(4), 159-177. http://dx.doi.org/10.1177/1524838010378299

Liberati, A., Altman, D. G., Tetzlaff, J., Mulrow, C., Gøtzsche, P. C., Ioannidis, J. P., Clarke, M., Devereaux, P. J., Kleijnen, J., \& Moher, D. (2009). The PRISMA statement for reporting systematic reviews and meta-analyses of studies that evaluate health care interventions: explanation and elaboration. Journal of clinical epidemiology, 62(10), 1 - 34. https://doi.org/10.1136/bmj.b2700

Maliszewski, G., \& Brown, C. (2014). Familism, substance abuse, and sexual risk among foster care alumni. Children and Youth Services Review, 36, 206 - 212. https://doi.org/10.1016/j.childyouth.2013.11.021

Oshima, K. M. M., Narendorf, S. C., \& McMillen, J. C. (2013). Pregnancy risk among older youth transitioning out of foster care. Children and Youth Services Review, 35(10), 1760 - 1765. https://doi.org/10.1016/j.childyouth.2013.08.001

Paolucci, E. 0., Genuis, M. L., \& Violato, C. (2001). A meta-analysis of the published research on the effects of child sexual abuse. The Journal of Psychology, 135(1), 17-36. http://dx.doi.org/10.1080/00223980109603677.

Pergamit, M. R., \& Johnson, H. (2009). Extending foster care to age 21: Implications and estimates from youth aging out of foster care in Los Angeles. Washington, DC: Urban Institute.

PettyJohn, M. E., Reid, T. A., Miller, E., Bogen, K. W., \& McCauley, H. L. (2021). Reproductive coercion, intimate partner violence, and pregnancy risk among adolescent women with a history of foster care involvement. Children and youth services review, 120. https://doi.org/10.1016/j.childyouth.2020.105731

Polgar, M., \& Auslander,W. (2009). HIV prevention for youths in foster care: Understanding future orientation and intended risk behaviors. Journal of HIV/AIDS \& Social Services, 8(4), 397-413. http://dx.doi.org/10.1080/15381500903417646

Ramseyer Winter, V., Brandon-Friedman, R. A., \& Ely, G. E. (2016). Sexual health behaviors and outcomes among current and former foster youth: A review of the literature. Children and Youth Services Review, 64, 1-14. https://doi.org/10.1016/j.childyouth.2016.02.023

Robertson, R. D. (2013). The invisibility of adolescent sexual development in foster care: Seriously addressing sexually transmitted infections and access to services. Children and Youth Services Review, 35(3), 493-504. http://dx.doi.org/10.1016/j.childyouth.2012.12.009

Satterwhite, C. L., Torrone, E., Meites, E., Dunne, E. F., Mahajan, R., Ocfemia, M. C. B., John, S., Fujie, X., \& Weinstock, H. (2013). Sexually transmitted infections among US women and men: Prevalence and incidence estimates, 2008. Sexually Transmitted Diseases, 40(3), 187-193.

http://dx.doi.org/10.1097/0LQ.0b013e318286bb53

Simkiss, D. E., Stallard, N., \& Thorogood, M. (2012). A systematic literature review of the risk factors associated with children entering public care. Child: Care, Health and Development, 39(5), 628-642. https://doi.org/10.1111/cch.12010

Stott, T. (2012). Placement instability and risky behaviors of youth aging out of foster care. Child and Adolescent Social Work Journal, 29(1), 61-83. http://dx.doi.org/10.1007/s10560-011-0247-8.

Surratt, H. L., \& Kurtz, S. P. (2012). Foster care history and HIV infection among drug-using African American female sex workers. AIDS and Behavior, 16(4), 982-989. http://dx.doi.org/10.1007/s10461-011-0008-x

Surratt, H. L., Inciardi, J. A., Kurtz, S. P., \& Kiley, M. C. (2004). Sex work and drug use in a subculture of violence. Crime \& Delinquency, 50(1), 43-59. http://dx.doi.org/10.1177/0011128703258875

Tarren-Sweeney, M. (2008). Predictors of problematic sexual behavior among children with complex maltreatment 
histories. Child Maltreatment, 13(2), 182 - 198. https://doi.org/10.1177=1077559508316043

Taussig, H. N. (2002). Risk behaviors in maltreated youth placed in foster care: A longitudinal study of protective and vulnerability factors. Child Abuse \& Neglect, 26(11), 1179-1199. http://dx.doi.org/10.1016/S01452134(02)00391-5

Taussig, H. N., \& Talmi, A. (2001). Ethnic differences in risk behaviors and related psychosocial variables among a cohort of maltreated adolescents in foster care. Child Maltreatment, 6(2), 180-192. http://dx.doi.org/10.1177/1077559501006002010

Thompson, R. G., \& Auslander,W. F. (2011). Substance use andmental health problems as predictors of HIV sexual risk behaviors among adolescents in foster care. Health \& Social Work, 36(1), 33 - 43. http://dx.doi.org/10.1093/hsw/36.1.33

Van Roode, T., Dickson, N., Herbison, P., \& Paul, C. (2009). Child sexual abuse and persistence of risky sexual behaviors and negative sexual outcomes over adulthood: Findings from a birth cohort. Child Abuse \& Neglect, 33(3), 161-172. http://dx.doi.org/10.1016/j.chiabu.2008.09.006

Voisin, D. R., \& Bird, J. (2009). What African American male adolescents are telling us about HIV infection among their peers: Cultural approaches for HIV prevention. Social Work, 54(3), 201-210. Retrieved from http://www.ncbi.nlm.nih.gov/pubmed/19530567

Wekerle, C., Goldstein, A. L., Tanaka, M., \& Tonmyr, L. (2017). Childhood sexual abuse, sexual motives, and adolescent sexual risk-taking among males and females receiving child welfare services. Child Abuse \& Neglect, 66, 101-111. http://dx.doi.org/10.1016/j.chiabu.2017.01.013

Wilson, E., Casanueva, C., Smith, K. R., Koo, H., Tueller, S. J., \& Webb, M. B. (2014). Risk of early sexual initiation and pregnancy among youth reported to the child welfare system. Child Welfare, 93(1), 127-147. 
